\title{
AHPND BIOMARKER PROFILE IDENTIFIED BY TRANSCRIPTOME SEQUENCING IN LITOPENAEUS VANNAMEI STOMACH
}

\author{
Tze Hann Ng, Chia-Wei Lu, Shih-Shun Lin, Chu-Fang Lo, Han-Ching Wang ${ }^{\S}$ \\ Institute of Biotechnology, National Cheng Kung University, Taiwan R.O.C.
}

\begin{abstract}
Acute hepatopancreatic necrosis disease (AHPND) is an emerging (since 2009) disease affecting pacific white shrimp (Litopenaeus vannamei) in Southeast Asia. It is generally accepted that it is caused by Vibro parahaemolyticus (VP), with extra-chromosomal elements that produce a virulent toxin. It was reported that VP may multiply in the shrimp stomach and cause severe cellular damage by releasing virulent toxin into shrimp hepatopancreas. In our pioneering studies, we hypothesized that severe cellular damage may also be due to immune overexpression following VP invasion. In addition to development of therapeutic approaches for AHPND, there is a need to monitor biomarkers of disease progression and host immune responses. In this study, next-generation sequencing-based transcriptome analysis of total RNA from shrimp stomach was performed. A non-virulent VP (S02) strain was used to normalize general responses after bacterial invasion. Shrimp were immersed in a virulent VP (5HP) strain and samples collected at 24 hours post immersion (hpi) and designated AHPND-infected shrimp. Furthermore, samples collected at 1 week post immersion (wpi) were designated AHPND-survived shrimp. For 24 hpi AHPND-infected shrimp, there were a few shrimp with no detectable AHPND toxin and immune overexpression, whereas for 1 wpi AHPND-survived shrimp, all lacked any detectable of AHPND toxin and immune overexpression. Therefore, we inferred that these shrimp may have triggered critical responses that can overcome VP invasion. By comparing these shrimp with severe AHPND-infected shrimp, we identified relative genes and pathways associated with AHPND pathogenesis and defenses. This study documented host gene changes in response to AHPND; furthermore, pathways identified may be key for successfully controlling AHPND.
\end{abstract}

\section{KEYWORDS}

Acute hepatopancreatic necrosis disease, AHPND, shrimp, transcriptome, Vibro parahaemolyticus

${ }^{\S}$ Corresponding author. Tel: +88662757575 ext 65603-810; Fax: +88662766505

E-mail address: wanghc@mail.ncku.edu.tw 